\title{
Phenotypic characterization and genomic DNA polymorphisms of Escherichia coli strains isolated as the sole micro-organism from vaginal infections
}

\author{
Olga Lobos and Carlos Padilla \\ Departamento de Microbiología, Universidad de Talca, Chile
}

Correspondence

Olga Lobos

olobos@utalca.cl

Received 4 August 2008

Revised 4 November 2008

Accepted 14 November 2008

\begin{abstract}
Vaginal infections such as vulvovaginal candiadiasis, trichomoniasis and bacterial vaginosis are common worldwide. Accurate diagnosis and prescription of appropriate treatments are important since these infections are linked to adverse outcomes for women during pregnancy and for newborns. Several aetiological agents are responsible for these infectious diseases; however, the presence of Escherichia coli in these infections is controversial. Thus, it is important to identify some phenotypic and genotypic properties of $E$. coli strains isolated from vaginal infections. Fortysix $E$. coli strains isolated from vaginal fluid as the sole micro-organism, and 20 other $E$. coli strains isolated from other samples (urinary tract infections, otitis and septicaemia) were analysed by several phenotypic tests. In addition, genotypic features were studied by RAPD-PCR techniques. Biochemical tests showed that the $E$. coli strains isolated from vaginal fluid could be grouped into a single cluster which is subdivided into two phenogroups. Analysis of the dendrogram based on fragment length polymorphisms of genomic DNA indicated that $E$. coli isolates from vaginal infections form a single cluster with two subdivisions. Further studies are needed to analyse the molecular structure and virulence characteristics of these $E$. coli strains in order to determine their potential role in vaginal infections.
\end{abstract}

\section{INTRODUCTION}

The vagina is a complex habitat for micro-organisms, and vaginal infections are considered to be among the main causes of morbidity around the world. Studies of the microbial flora of the human vagina reveal that microorganisms normally present in this habitat play an important role in preventing colonization by exogenous bacterial species (Donders et al., 2000; van De Wijgert et al., 2000). The vaginal microflora is usually constituted by 5-15 different bacterial species (Obata-Yasuoka et al., 2002), normally characterized by a high number of Lactobacillus species. Lactobacillus crispatus and Lactobacillus jensenii are of great importance in maintaining the vaginal $\mathrm{pH}$ at approximately 4.7 (or less), which causes growth inhibition of other micro-organisms (Marrazzo, 2003).

Three major types of infections that affect the vaginal habitat are vulvovaginal candiadiasis, trichomoniasis and bacterial vaginosis. The first two have been extensively characterized in the literature (Marrazzo, 2003). Bacterial vaginosis is the most common cause of vaginal discharge (Klebanoff et al., 2004) and is described as a disequilibrium in the vaginal microflora in which the normally predom-

Abbreviations: RAPD, random amplified polymorphic DNA; UTI, urinary tract infection(s). inant Lactobacillus species are overgrown by anaerobic and facultative species (Ness et al., 2005). This condition is not associated with vaginal inflammation as occurs in vaginitis. Vaginal infections, but principally bacterial vaginosis, have been associated with many complications, including second trimester miscarriage, pelvic inflammatory disease, preterm birth, preterm premature rupture of the membranes, chorioamnionitis, post-partum endometritis, postoperative infection after gynaecological surgery and easier acquisition of HIV (Beigi et al., 2004; Ness et al., 2004). The aetiological agents of vaginal infection are Candida albicans in vulvoginal candidiasis, and Trichomonas vaginalis in trichomoniasis. The predominant microorganisms that cause bacterial vaginosis are Gardnerella vaginalis, Mycoplasma hominis, Ureaplasma urealyticum and others such as Prevotella spp., Mobiluncus spp. and Bacteroides spp. (Klebanoff et al., 2004).

Escherichia coli is a commensal inhabitant of the intestinal tract of healthy humans and different animals (Clermont et al., 2000). It is accepted that pathogenic E. coli strains are derived from commensal strains by acquisition of chromosomal or extrachromosomal virulence operons, and although extraintestinal pathogenic E. coli strains are mostly opportunistic, they can cause a diverse spectrum of serious diseases (Escobar-Páramo et al., 2004; Sasakawa \& Hacker, 2006). The presence of vaginal E. coli is 
controversial with regard to its potential pathogenic role. Some studies report its presence as an opportunistic microorganism (Watt et al., 2003). Others concluded that this species has not been detected on healthy vaginal epithelium (Hyman et al., 2005), and also it has been isolated from aerobic vaginitis. This infection is a condition of abnormal vaginal flora that differs from bacterial vaginosis. Genital complaints are those of a real vaginitis. Group B streptococci, E. coli, Staphylococcus aureus and T. vaginalis are frequently cultured (Donders et al., 2002). Considering the serious obstetric and neonatal complications, and that about $30 \%$ of women with vaginal complaints remain undiagnosed despite extensive testing (Anderson et al., 2004), it is of great importance to determine whether E. coli strains isolated as the sole micro-organism from vaginal infections have particular characteristics. Thus, the aims of this study were to identify E. coli strains isolated as the sole micro-organism from women of reproductive age and presenting vaginal infections, to analyse their phenotypic characteristics by means of biochemical tests, and to determine potential genotypic association among them. E. coli strains isolated in association with other known aetiological agents of vaginal infections were not considered in the present study.

\section{METHODS}

Study population. The study population was composed of 425 women of fertile age (18 to 41 years) who attended Talca City Hospital with clinical diagnosis of vaginal infection; they were nonpregnant, non-smokers, had no history of sexually transmitted infections, and had had one sexual partner during the previous 6 months. Also, they had had no antimicrobial treatment within the previous 6 months as revealed by a written questionnaire. In addition, they showed absence of menstruation and had no diarrhoea or urinary tract infections (UTI). Diagnosis of vaginosis was based on detection of three or more Amsel criteria (Amsel et al., 1983) and the microscopic record of bacterial morphology to detect bacterial vaginosis (Nugent et al., 1991). The study protocol was accepted by the Universidad de Talca Bioethical Committee and included an informed consent from the patients, before collecting clinical samples. As control samples, vaginal epithelium from 100 healthy women was analysed by microbiological procedures.

Sample collection and microbiology study. Vaginal swabs (three swabs per patient) were taken from the lateral and posterior vaginal fornix. One was immediately streaked on Brucella agar (Difco) supplemented with sheep blood $(5 \%)$, vitamin $\mathrm{K}\left(1 \mu \mathrm{g} \mathrm{ml} \mathrm{ml}^{-1}\right)$ (Merck), haemin $\left(5 \mu \mathrm{g} \mathrm{ml}^{-1}\right)$ (Merck) and kanamycin $\left(50 \mu \mathrm{g} \mathrm{ml}^{-1}\right)$ (Sigma) with the purpose of obtaining growth of Bacteroides fragilis, Fusobacterium nucleatum, Porphyromonas gingivalis and Prevotella intermedia. Plates were incubated at $37{ }^{\circ} \mathrm{C}$ for 12 days in an anaerobic system (bioMérieux). The same swab was shaken into tubes with Schaedler broth (BD) and incubated anaerobically at $37{ }^{\circ} \mathrm{C}$ for 7 days to demonstrate growth of Mobiluncus spp. A second swab was streaked onto MacConkey agar (Difco) and Trypticase Soy agar (Difco) supplemented with sheep blood (5\%) and incubated in aerobic conditions at $37^{\circ} \mathrm{C}$ for $24 \mathrm{~h}$. The same swab was streaked onto Thayer Martin agar (Difco) and incubated in the presence of $\mathrm{CO}_{2}(5 \%)$ at $37{ }^{\circ} \mathrm{C}$ for $48 \mathrm{~h}$. Finally, the swabs were used to inoculate a Sabouraud agar plate (Merck) and on Trichomonas medium (Oxoid), for Trichomonas vaginalis growth. The third swab was used with the Mycoplasma IST-2 kit (bioMérieux) to detect the growth of Mycoplasma hominis and Ureaplasma urealyticum. Finally the same swab was used to detect Chlamydia trachomatis with the TestPack Chlamydia kit (Abbot). Identification of Enterobacteriaceae family members was done with the API 20-E test (bioMérieux) and anaerobic bacteria were identified by phenotypic methods (Jousimes-Somer \& Sumanen, 1999). Candida albicans was identified by the ID 32C kit (bioMérieux).

In order to compare phenotypic and genotypic characteristics of vaginal $E$. coli strains with other E. coli isolates from other origins, 15 E. coli isolates from non-pregnant women with UTI, and four E. coli isolates from blood samples of patients with septicaemia and one from a patient with otitis were used. These isolates, obtained with informed consent from patients (as described above), were identified by the same procedures as used with isolates from vaginal infections.

Determination of DNA polymorphism in E. coli strains isolated from vaginal infections by RAPD-PCR. Phylogenetic relationships among the E. coli strains isolated from vaginal infections and those used to compare phenotypic and genotypic characteristics were studied by means of random amplified polymorphic DNA (RAPD). This method has become a highly valuable molecular tool for intraspecies characterization of E. coli (Cave et al., 1994; Wang et al., 1993). Genomic DNA was extracted from a single colony using the Aqua Pure Genomic DNA Isolation kit (Bio-Rad) and the DNA concentration was spectrophotometrically determined $\left(A_{260}\right)$. PCRs were conducted in $25 \mu \mathrm{l}$ reaction mixture containing $25 \mathrm{ng}$ DNA template, $1 \mathrm{U}$ Taq DNA polymerase (Invitrogen) in $1 \times$ buffer, $300 \mu \mathrm{M}$ of each dNTP and $0.8 \mathrm{nM}$ of each primer. The primers used were $2 \mathrm{H}$ (5'-AAGCTTCGACTGT- $\left.3^{\prime}\right)$ and $3 \mathrm{H}$ (5'-AAGCTTGATTGCCC-3), which were randomly selected among four arbitrary primers. Amplification was done in a DNA Engine (Bio-Rad) thermal cycler programmed to one cycle of $5 \mathrm{~min}$ at $94^{\circ} \mathrm{C}, 10$ cycles of $1 \mathrm{~min}$ at $94{ }^{\circ} \mathrm{C}, 1 \mathrm{~min}$ at $36{ }^{\circ} \mathrm{C}$, and $2 \mathrm{~min}$ at $72{ }^{\circ} \mathrm{C}$ followed by 20 cycles of $1 \mathrm{~min}$ at $94{ }^{\circ} \mathrm{C}, 1 \mathrm{~min}$ at $50{ }^{\circ} \mathrm{C}$ and $2 \mathrm{~min}$ at $72{ }^{\circ} \mathrm{C}$ to allow the completion of DNA extension (Aslam et al., 2004). A negative control, consisting of the same mixture but using water instead of DNA, was included in each run. This procedure was repeated twice per strain. Only fragments present in both amplifications were used to define the RAPD patterns. The amplification products were analysed by electrophoresis in $1 \%$ agarose gel, stained with ethidium bromide $\left(0.5 \mu \mathrm{g} \mathrm{ml}^{-1}\right)$ and photographed under UV light. A $1 \mathrm{~kb}$ DNA ladder (New England Biolabs) was included as molecular size marker. The RAPD patterns obtained from E. coli strains isolated from vaginal infections were compared to those obtained from E. coli strains isolated from UTI, septicaemia and otitis. The numerical analysis was carried out using Taxotron software (Taxolab) (Chatellier et al., 1997). For each strain, the RAPD type was defined as the combination of the band pattern obtained with both primers. The relationships among the RAPD types of the strains were calculated by the unweighted pair-group method averages method and represented as a dendrogram.

Statistical analysis. Significance of differences between variables was tested by means of $\chi^{2}$ test and Fisher's exact test.

\section{RESULTS}

\section{Bacteriological analysis of vaginal fluids}

Table 1 shows that Gardnerella vaginalis was the most frequent micro-organism isolated $(33.2 \%)$ followed by $E$. coli $(23.0 \%)$ in the 425 women with clinical diagnosis of vaginal infection. Other micro-organisms frequently 
Table 1. Micro-organisms isolated from women with vaginal infections and from the vagina of healthy women

\begin{tabular}{|lcccc|}
\hline \multirow{2}{*}{ Micro-organism } & \multicolumn{3}{c|}{ Vaginal infections } & Healthy women $\boldsymbol{n}(\%)$ \\
\cline { 2 - 4 } & Total $\boldsymbol{n}(\%)$ & Vaginitis $\boldsymbol{n}(\%)$ & Vaginosis $\boldsymbol{n}(\%)$ & \\
\hline G. vaginalis & $141(33.2)$ & $12(2.8)$ & $129(30.3)$ & $26(26)$ \\
E. coli & $96(23.0)$ & $27(6.3)$ & $69(16.2)$ & $6(6)$ \\
C. albicans & $66(15.5)$ & $66(15.5)$ & 0 & 0 \\
T. vaginalis & $39(9.1)$ & $39(9.1)$ & 0 & $6(6)$ \\
Mobiluncus spp. & $12(2.8)$ & 0 & $12(2.8)$ & $2(2)$ \\
Ent. faecalis & $10(2.4)$ & 0 & $10(2.4)$ & 0 \\
GNAB & $9(2.2)$ & 0 & $9(2.2)$ & 0 \\
M. hominis & $5(1.2)$ & 0 & $5(1.2)$ & $8(8)$ \\
C. trachomatis & $2(0.5)$ & $2(0.5)$ & $3(0.7)$ & $56(56)$ \\
S. agalactiae & $3(0.7)$ & 0 & $28(6.6)$ & $100(100)$ \\
NGM $\dagger$ & $42(9.9)$ & $14(3.3)$ & $265(62.4)$ & \\
Total & $425(100)$ & $160(37.5)$ & & \\
\hline
\end{tabular}

${ }^{*} \mathrm{GNAB}$, Gram-negative strictly anerobic bacilli (B. fragilis, P. gingivalis, P. intermedia, F. nucleatum).

$\dagger$ NGM, No growth of micro-organisms, but existing growth of Lactobacillus spp. and coagulase-negative Staphylococcus.

isolated were Candida albicans and Trichomonas vaginalis, $15.5 \%$ and $9.1 \%$, respectively. Gram-negative strictly anaerobic bacilli were isolated in only $2.2 \%$ of the cases. No growth of Neisseria gonorrhoeae or Ureaplasma urealyticum was observed. Moreover, in 42 of the samples studied no bacterial growth was detected. Also, 26 vaginal samples from healthy women yielded $G$. vaginalis strains and a low growth of E. coli, Mobiluncus spp., Enterococcus faecalis, Streptococcus agalactiae and Gram-negative strictly anaerobic bacilli (Bacteroides fragilis, Porphyromonas gingivalis, Prevotella intermedia and Fusobacterium nucleatum). Table 2 shows micro-organisms recovered from women with vaginal infection. E. coli strains appeared associated with G. vaginalis, C. albicans and T. vaginalis in 29, 12 and 8 samples of vaginal fluid respectively. Individually, $G$. vaginalis was the most frequently isolated micro-organism, from 97 samples, and E. coli was isolated as a single microorganism in 46 samples.

\section{Phenotypic properties of the E. coli strains}

A comparison of the biochemical test results obtained for E. coli isolates from vaginal specimens and from other sources is shown in Table 3. Tests for ornithine decarboxylase $(P \leqslant 0.001)$, L-rhamnose $(P \leqslant 0.022)$ and sucrose fermentation $(P \leqslant 0.0001)$ showed significant differences between the two groups of E. coli strains.

\section{RAPD analysis of $E$. coli strains isolated from vaginal fluid as the sole micro-organism}

RAPD analysis of genomic DNA of the 46 E. coli strains isolated as the sole micro-organism from vaginal samples resulted in 63 profiles when using primers $2 \mathrm{H}$ and $3 \mathrm{H}$. A profile of ten and seven polymorphic fragments was observed with the $3 \mathrm{H}$ and $2 \mathrm{H}$ primer set, respectively.
The molecular masses of the fragments obtained with the $3 \mathrm{H}$ primer ranged between 500 and $4500 \mathrm{bp}$ and the fragments produced by the $2 \mathrm{H}$ primer ranged between 500 and $6000 \mathrm{bp}$ (data not shown). A total of 46 RAPD profiles were observed among vaginal isolates and 17 among isolates from other sources. The dendrogram presented in Fig. 1 shows three main clusters, labelled A, B and C. Cluster A included a single isolate from otitis. Cluster B encompassed the $46 \mathrm{E}$. coli strains of vaginal origin plus four strains from septicaemia, and cluster $\mathrm{C}$ included the

Table 2. Number of samples from women with vaginal infection that yielded growth of associated and individual micro-organisms.

\begin{tabular}{|lc|}
\hline Micro-organism(s) & No. of samples \\
\hline G. vaginalis + E. coli & 29 \\
C. albicans + E. coli & 12 \\
T. vaginalis + E. coli & 8 \\
G. vaginalis + Mobiluncus spp. & 6 \\
G. vaginalis + C. albicans & 5 \\
G. vaginalis + GNAB & 4 \\
G. vaginalis & 97 \\
C. albicans & 49 \\
E. coli & 46 \\
T. vaginalis & 31 \\
E. faecalis & 10 \\
Mobiluncus spp. & 6 \\
M. hominis & 5 \\
GNAB & 5 \\
S. agalactiae & 3 \\
C. trachomatis & 2 \\
\hline
\end{tabular}

${ }^{*}$ GNAB, Gram-negative strictly anerobic bacilli (B. fragilis, $P$. gingivalis, P. intermedia, F. nucleatum). 
Table 3. Phenotypic characteristics of E. coli strains isolated from women with vaginal infections as the sole micro-organism in comparison with $E$. coli strains from other sources

Tests positive for all strains: ONPG, indole production, D-glucose acid, D-mannitol fermentation, D-sorbitol fermentation, D-arabitol fermentation, nitrate to nitrite and lysine decarboxylase. Tests negative for all strains: citrate (Simmons), hydrogen sulfide, urea hydrolysis, tryptophan deaminase, Voges-Proskauer (acetoin), gelatin hydrolysis and oxidase.

\begin{tabular}{|lccccc|}
\hline Biochemical test & \multicolumn{3}{c|}{ E. coli strains } \\
\cline { 2 - 3 } & $\begin{array}{c}\text { Vaginal source } \\
(\boldsymbol{n}=\mathbf{4 6 )}\end{array}$ & & $\begin{array}{c}\text { Other source } \\
(\boldsymbol{n}=\mathbf{2 0})\end{array}$ \\
\cline { 2 - 3 } \cline { 5 - 6 } & No. & $(\%)$ & & No. & $(\%)$ \\
\hline Melibiose fermentation & 45 & $(97)$ & & 20 & $(100)$ \\
L-Rhamnose fermentation & 44 & $(95)$ & & 15 & $(75)$ \\
Ornithine decarboxylase & 29 & $(63)$ & & 20 & $(100)$ \\
Sucrose fermentation & 24 & $(52)$ & & 20 & $(100)$ \\
Arginine dihydrolase & 16 & $(34)$ & & $(15)$ \\
Amygdalin fermentation & 2 & $(4)$ & & $(0)$ \\
myo-Inositol fermentation & 1 & $(2)$ & 0 & $(0)$ \\
& & & & & \\
\hline
\end{tabular}

isolates from UTI. The B cluster was subdivided into two genogroups (B1, 23 strains, and B2, 20 strains), both with E. coli strains of vaginal origin. Genogroups B1 and B2 represented $93.5 \%$ of all $E$. coli strains obtained from a vaginal source.

\section{DISCUSSION}

Vaginal infections are still a major cause of morbidity, leading to millions of dollars spent worldwide annually in physician services and medications. Vaginal infections are not reportable diseases, and therefore accurate estimates of incidence are unavailable (Schwebke, 2000). Infections usually respond to appropriate treatments; however misdiagnosis and pharmacological failure may occur. Therefore, it is important to diagnose the problem accurately and prescribe the appropriate treatment (Owen \& Clenney, 2004). The high prevalence of vaginal infections, along with controversies with regard to aetiological agents, may be related to unidentified bacteria as agents of such infections. The present study establishes differences among E. coli strains isolated from different human habitats, including the vagina, and identifies an association between strains isolated as the sole microorganism from women with vaginal infections with regard to both phenotypic and genotypic properties. This finding supports a potential role of particular subgroups of E. coli in the pathogenesis of vaginal infections.

The aetiological role of E. coli in vaginal infections has been controversial. In general, their isolation from vaginal fluids is considered as normal, and women are not treated. Our study of vaginal specimens from 425 women with clinical diagnosis of vaginal infection showed $62.4 \%$ of bacterial vaginosis cases in comparison to $37.5 \%$ of vaginitis, in agreement with previous reports (Klebanoff et al., 2004; Beigi et al., 2004). Moreover, it is important to observe that $T$. vaginalis and $C$. albicans were restricted to vaginitis infection. E. coli was present in both bacterial vaginosis and vaginitis (23\% of total bacterial isolates), which made it interesting to determine if $E$. coli strains can be associated with other aetiological agents. In this study E. coli was isolated as the sole micro-organism in 46 cases. The absence of other recognized aetiological agents of vaginal infection made these bacterial strains an interesting research topic.

When the strains from vaginal, UTI, septicaemia and otitis sources were analysed for ornithine decarboxylase, sucrose fermentation and rhamnose fermentation, only those isolated as the sole micro-organism from vaginal infections showed significant particular activities. Thus, only $63 \%$ of these E. coli strains showed positive activity for ornithine decarboxylase, and $95 \%$ and $52 \%$ showed positive activity on rhamnose and sucrose, respectively (versus $100 \%, 75 \%$ and $100 \%$, respectively, for the strains from other sources). It is important to note that phenotypic and chemotaxonomic characteristics are indirect markers of genomic changes (Coenye et al., 2005).

Results obtained by means of RAPD showed different profiles of genomic DNA. These molecular profiles were highly reproducible, allowing the analysis of different genotypic profiles by means of a dendrogram. The results showed that the E. coli strains grouped in three clusters. The strains of vaginal origin were clustered together in a distinct cluster, subdivided into two genogroups, which constituted $93.5 \%$ of the total vaginal isolates. This suggests that these strains, isolated as the sole microorganism from vaginal infections, constitute a separate subpopulation within the species. Our study further demonstrated that E. coli strains isolated from UTI are closely related to the strains of vaginal origin. Other studies have suggested that $E$. coli vaginal residents present a common phylogeny to uropathogenic E. coli strains (Obata-Yasuoka et al., 2002) and also that uropathogenic E. coli are better adapted than other E. coli to the urethra, periurethra and vagina (Foxman et al., 2002). It is possible that in the referenced studies $E$. coli was not isolated as the sole micro-organism from vaginal infections and was not causally associated with the vaginal infection.

According to our results, it is plausible to postulate that the E. coli strains isolated as the sole micro-organism from the vagina may belong to a particular phylogenetic group. This could be based on the high genomic plasticity of E. coli and its capacity to acquire or eliminate genes. Since microorganisms are defined by their environment, it would be unlikely that micro-organisms capable of causing human diseases evolve in such an environment where there are no humans or other animals (Wilson \& Salyers, 2003). This is 


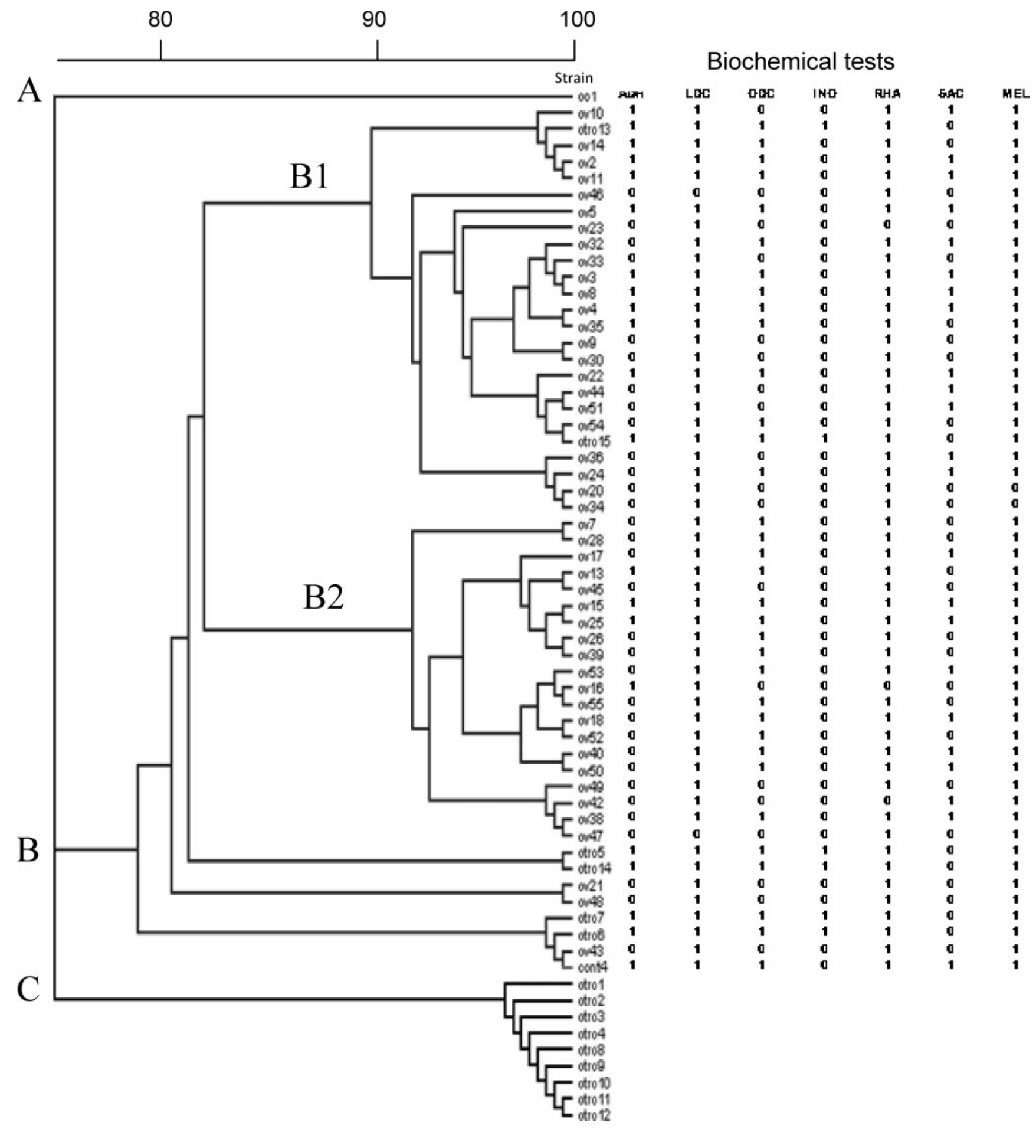

Fig. 1. Genetic relationships among the $E$. coli strains studied and the respective biochemical tests used for vaginal $E$. coli $(0$, negative biochemical test; 1 , positive biochemical test). ADH, arginine dihydrolase; LDC, lysine decarboxylase; ODC, ornithine decarboxylase; INO, myo-inositol fermentation; RHA, L-rhamnose fermentation; SAC, sucrose fermentation; MEL, melibiose fermentation. 
likely to apply with respect to mutations that lead to increasing virulence, considering that the amplification of those mutations requires a selective pressure, presumably provided by the host environment, the vagina habitat in this case.

Many genital infections occur as a result of new sexual practices, involving significant microbiological aspects. This could explain the presence of new aetiological agents of vaginal infections or the adaptation of micro-organisms such as E. coli. Finally, the potential role of E. coli in vaginal infections deserves further molecular studies to learn more about the particular subset of this species that appears to be aetiologically involved.

\section{ACKNOWLEDGEMENTS}

Technical assistance from Claudio Ramirez and Fernando Poblete was invaluable. Financial support for this study was provided by Grant DI 280, Dirección de Investigación, Universidad de Talca.

\section{REFERENCES}

Amsel, R., Totten, P. \& Spiegel, C. A. (1983). Non-specific vaginitis: diagnostic criteria and microbial and epidemiological associations. Am J Med 74, 14-22.

Anderson, M. R., Klink, K. \& Cohrssen, A. (2004). Evaluation of vaginal complaints. JAMA 291, 1368-1379.

Aslam, M., Greer, G., Nattres, F., Gill, C. \& McMullen, L. (2004). Genetic diversity of Escherichia coli recovered from the oral cavity of beef cattle and their reladness to faecal Escherichia coli. Lett Appl Microbiol 39, 523-527.

Beigi, R. H., Austin, M. N., Meyn, L. A., Krohn, M. A. \& Hillier, S. L. (2004). Antimicrobial resistance associated with the treatment of bacterial vaginosis. Am J Obstet Gynecol 191, 1124-1129.

Cave, H., Bingen, J. E. \& Denamur, E. (1994). Differentiation of Escherichia coli strains using randomly amplified polymorphic DNA analysis. Res Microbiol 145, 141-150.

Chatellier, S., Ramanantsoa, C., Harriau, P., Rolland, K., Rosenau, A. \& Quenti, R. (1997). Characterization of Streptococcus agalactiae strains by a randomly amplified polymorphic DNA analysis. J Clin Microbiol 35, 2573-2579.

Clermont, O., Bonarcosi, S. \& Bingen, E. (2000). Rapid and simple determination of the Escherichia coli phylogenetic group. Appl Environ Microbiol 66, 4555-4558.

Coenye, T., Gevers, D., van de Peer, Y., Vandamme, P. \& Swings, J. (2005). Towards a prokaryotic genomic taxonomy. FEMS Microbiol Rev 29, 147-167.

Donders, G. G., Bosmans, E., Dekkermaecker, A., Verecken, A., Bulck, B. V. \& Splitz, B. (2000). Pathogenesis of abnormal vaginal bacterial flora. Am J Obstet Gynecol 182, 872-878.

Donders, G. G., Vereecken, A., Bosmans, E., Dekeersmaecker, A., Salembier, G. \& Spitz, B. (2002). Definition of a type of abnormal vaginal flora entity that is distinct from bacterial vaginosis: aerobic vaginitis. BJOG 109, 34-43.
Escobar-Páramo, P., Grenet, K., Le Menac'h, A., Rode, L., Salgado, E., Amorin, C., Gouriou, S., Picard, B., Andremont, A. \& other authors (2004). Large-scale population structure of human commensal Escherichia coli isolates. Appl Environ Microbiol 70, 5698-5700.

Foxman, B., Manning, S. D., Tallman, P., Bauer, R., Zhang, L., Koopman, J. S., Gillespie, B., Sobel, J. D. \& Marrs, C. F. (2002). Uropathogenic Escherichia coli are more likely than commensal E. coli to be shared between heterosexual sex partners. Am J Epidemiol 156, $1133-1140$.

Hyman, R. W., Fukushima, M., Diamond, L., Kumm, J., Giudice, L. C. \& Davis, R. W. (2005). Microbes on the human vaginal epithelium. Proc Natl Acad Sci U S A 102, 7952-7957.

Jousimes-Somer, H. \& Sumanen, P. (1999). Anaerobic Gram negative rods and cocci. In Manual of Clinical Microbiology, 7th edn, pp. 690-711. Edited by P. Murray, E. Baron, M. Pfaller, F. Tenover \& R. Yolken. Washington, DC: American Society for Microbiology.

Klebanoff, M. A., Schwebke, J. R., Zhang, J., Nansel, T. R., Yu, K. F. \& Andrews, W. W. (2004). Vulvovaginal symptoms in women with bacterial vaginosis. Obstet Gynecol 104, 267-272.

Marrazzo, J. M. (2003). Bacterial vaginosis. Curr Treat Opt Infect Dis 5, 63-68.

Ness, R. B., Hillier, S. L., Kip, K. E., Soper, D. E., Stamm, C. A. \& Mcgregor, J. A. (2004). Bacterial vaginosis and risk of pelvic inflammatory disease. Obstet Gynecol 104, 761-769.

Ness, R. B., Kip, K. E., Hillier, S. L., Soper, D. E., Stamm, C. A., Sweet, R. L., Rice, P. \& Richter, H. E. (2005). A cluster analysis of bacterial vaginosis-associated microflora and pelvic inflammatory disease. $\mathrm{Am} \mathrm{J}$ Epidemiol 162, 585-590.

Nugent, R. P., Krhon, M. A. \& Hillier, S. L. (1991). Realiability of diagnosing bacterial vaginosis is improved by a standardized method of Gram stain interpretation. J Clin Microbiol 29, 297-301.

Obata-Yasuoka, M., Ba-Thein, W., Hamada, H. \& Hayashi, H. (2002). A multiplex polymerase chain reaction-based diagnostic method for bacterial vaginosis. Obstet Gynecol 100, 759-764.

Owen, M. K. \& Clenney, T. L. (2004). Management of vaginitis. Am Fam Physician 70, 2125-2132.

Sasakawa, C. \& Hacker, J. (2006). Host-microbe interaction: bacteria. Curr Opin Microbiol 9, 1-4.

Schwebke, J. R. (2000). Bacterial vaginosis. Curr Infect Dis Rep 2, 14 17.

van De Wijgert, J. H., Mason, P. R., Gwanzura, L., Mbizvo, M. T., Chirenje, Z. M., Ilif, V., Shibosky, S. \& Padian, N. S. (2000). Intravaginal practices, vaginal flora disturbances, and acquisition of sexually transmitted diseases in Zimbabwean women. J Infect Dis 181, 587-594.

Wang, G., Whittman, T. S., Ber, C. M. \& Berg, D. E. (1993). RAPD (arbitrary primer) PCR is more sensitive than multilocus enzyme electrophoresis for distinguishing related bacterial strains. Nucleic Acids Res 21, 5930-5933.

Watt, S., Lanotte, P., Mereghetti, L., Moulin-Schouler, M., Picard, B. \& Quentin, R. (2003). Escherichia coli strains from pregnant women and neonates. Intraspecies genetic distribution and prevalence of virulence factors. J Clin Microbiol 41, 1929-1935.

Wilson, B. A. \& Salyers, A. A. (2003). Is the evolution of bacterial pathogens an out-of-body experience? Trends Microbiol 11, 347-350.

Edited by: M. Kilian 